\title{
Defective Particles Can Lead to Underestimated Antibody Titers in Virus Neutralization Tests
}

\author{
Wiebke Handke Detlev H. Krüger Andreas Rang \\ Institute of Virology, University Hospital Charité, Berlin, Germany
}

\section{Key Words}

Defective particles $\cdot$ Hantavirus $\cdot$ Focus reduction

neutralization test

\begin{abstract}
Objective: Stocks of RNA viruses often contain divergent mixtures of infectious and defective particles (DPs). Since the surface of both particles share the same immunoreactivity, differing ratios of these particles in stocks used for virus neutralization tests might alter the results. Here the impact of such off-target effects were measured. Methods: To determine the relative content of DPs in 3 stocks with high, medium and low titers of infectious virions, the amounts of nucleocapsid protein and viral RNA were quantified by Western blot and quantitative RT-PCR. Thereafter, stocks with different amount of DPs were used to determine the titer of neutralizing antibodies in serum from a convalescent Puumala virus-infected patient by a focus reduction neutralization test. Results: The relative amount of DPs was at least 5 -fold higher in the stock with the lowest titer compared to the stock with the highest. Stocks with lower levels of DPs led to higher antibody titers compared to the analysis performed with stocks containing higher levels of DPs. Conclusion: These results imply that DPs in virus stocks should be considered when performing virus neutralization tests to quantify neutralizing antibodies.

Copyright $\odot 2009$ S. Karger AG, Basel
\end{abstract}

\section{Introduction}

The genus Hantavirus belongs to the family Bunyaviridae, characterized by a tri-segmented single-stranded RNA genome. The natural hosts are mainly rodents in which the virus persists without obvious symptoms. Infection of humans, e.g. by aspiration of aerosolized excreta from infected animals, can lead to hemorrhagic fever with renal syndromes (HFRS) in Eurasia and hantavirus cardiopulmonary syndromes in America, depending on the hantavirus species involved. In Europe, most HFRS cases are caused by the Puumala virus (PUUV) transmitted by the bank vole (Myodes glareolus) and Dobrava-Belgrade virus transmitted by the yellow-necked mouse (Apodemusflavicollis), striped field mouse (Apodemus agrarius) or Caucasian wood mouse (Apodemus ponticus) $[1,2]$.

Viral RNA is generally detectable only in the early symptomatic phase of the disease caused by hantaviruses. After a decrease of the viral load, significant amounts of antibodies, including neutralizing antibodies, are detectable. To determine the causative agent, the specificity of such neutralizing antibodies can be typed by a focus reduction neutralization test (FRNT) or related assays. Due to cross-reactivity, a set of related hantavirus species is neutralized by antibodies in sera from hantavirus-infected patients. However, the reciprocal

\section{KARGER}

Fax +4161306 1234 E-Mail karger@karger.ch www.karger.com (c) 2009 S. Karger AG, Basel

$0300-5526 / 09 / 0526-0335 \$ 26.00 / 0$

Accessible online at:

www.karger.com/int
Andreas Rang

University Hospital Charité, Institute of Virology

Helmut-Ruska-Haus

DE-10098 Berlin (Germany)

Tel. +4930450 525 091, Fax +4930450525 907, E-Mail andreas.rang@ charite.de 
neutralizing end-point titer of a serum should be at least 4-fold higher for the causative agent compared to its neutralization capacity to other hantaviruses. This serologic difference represents 1 criterion for the definition of hantavirus species [3]. Based on neutralization assays with reference stocks, the virus species that yields the highest reciprocal neutralizing antibody titer is usually considered to be the pathogenic agent responsible for the disease.

For FRNT, only the amounts of infectious virions in the reference stocks are appointed, but the quantity of defective particles (DPs) is generally neglected $[4,5]$. It is well known that DPs contain a defective genome in an otherwise intact virion, which are generated in vivo and in vitro. Any gene whose product is deliverable in trans by the wild-type virus can be impaired, but not the regions required for replication and encapsidation of the genome. Since DPs are produced at the expense of the wild type, high levels of DPs decrease the yield of infectious virions. The ratio between both types of particles can vary significantly and the extent of this variation basically depends on the number of passages and multiplicity of infection $[6,7]$. DPs derived from cytolytic viruses can be removed by classical plaque purification. In accordance with such classical procedures, a focus purification method was recently established for noncytolytic viruses as well. In line with the theory that DPs reduce resources required for production of infectious virions, the titers of hantaviruses which had been subjected to this novel purification procedure increased 10 - to 1,000 fold [8].

Because defective and infectious virions share the same immunoreactivity, neutralizing antibodies should also react with DPs. The presented data demonstrate that increasing amounts of DPs in reference stocks used for virus neutralization assays can recruit neutralizing antibodies and correspondingly alter the results.

\section{Material and Methods}

\section{Cell Culture and Virus Production}

Vero cells (C1008, clone of Vero 76; LGC Promochem GmbH) were grown in Dulbecco's modified Eagle's medium supplemented with $5 \%$ fetal calf serum, $100 \mathrm{IU}$ penicillin, and $100 \mu \mathrm{g} / \mathrm{ml}$ streptomycin at $37^{\circ}$ in $5 \% \mathrm{CO}_{2}$. The PUUV strain Sotkamo (and Hantaan virus (HTNV) 76-118) was kindly provided by Dr. A. Lundkvist. Cells were infected and the culture medium was harvested and stored at $-80^{\circ} 7$ days later. Stocks designated PUUVhigh and PUUV-medium (according to the titer) derived from virus material which had been subjected to focus purification, performed as recently described [8]. Briefly, Vero cells grown in 6-well plates were infected by different dilutions of PUUV for $1 \mathrm{~h}$ at $37^{\circ}$, fed with medium containing $0.5 \%$ agarose and incubated for 10 days. After preincubation of the plate at $4^{\circ}$ for $30 \mathrm{~min}$, the medium/agarose overlays were engrailed at the $6 \mathrm{o}^{\prime} \mathrm{clock}$ position to mark the orientation, lifted off, transferred to Petri dishes and stored at $4^{\circ}$ as a source for subsequent recovery of the virus. The cells of the plate were washed with PBS and fixed with methanol for $10 \mathrm{~min}$. Virus-infected cells were detected by immunochemistry with a nucleocapsid protein (N protein)-specific antiserum. Medium/agarose overlays from wells with 1-5 foci were selected to recover infectious virus clones. A hard copy of the image showing the relative location of infected cells on the wells was used to pinpoint and recover infectious virions diffused into the corresponding region of the agarose overlay. Infectious virus was picked from the agarose, mixed vigorously in $200 \mu \mathrm{l}$ of medium and propagated in Vero cells. The titers of virus stocks were quantified accordingly and expressed as focus forming units (FFU) per milliliter [4].

\section{RNA Isolation and Quantitative RT-PCR}

Viral RNA was isolated from the PUUV stocks with a QiAamp Viral RNA Isolation Kit (Qiagen GmbH, Hilden, Germany) according to the manufacturer's instructions. Prior to RNA isolation, defined amounts of HTNV (17,000 FFU) were spiked to each sample to control recovery. About $10 \%$ of the isolated RNA was reverse-transcribed using random hexamer primers and Moloney murine leukemia virus reverse transcriptase (Invitrogen, Karlsruhe, Germany) for $1 \mathrm{~h}$ at $37^{\circ}$ following the manufacturer's recommendations. PUUV and HTNV cDNA was quantified by real-time PCR in a LightCycler-1 with species-specific primers and the FastStart DNA Master SYBR ${ }^{\circledR}$ Green Kit (Roche). The following PUUV- and HTNV-specific primer pairs were used: PUU-L6269f 5' -AGC AGT TAT CTC TAA TGG-3', PUUL6521r 5'-ATA AGA AGT ATA ATC TGT TAA-3', HTN-L6256f 5'-CAA ACA GGT CTC ATT ATT AG-3', and HTN-L6458r 5'CCT CCT CAA CAG TAC CAT-3'. Primer design was based on PUUV and HTNV sequences with accession numbers NC_ 005225 and NC_005222, respectively. The relative amount of viral RNA in 2 stocks was calculated with the formula: $\mathrm{n}$-fold amount of RNA in sample A versus sample B $=E($ ct A - ct B). E represents the PCR efficiency, determined by amplification of serial dilutions of the viral RNAs. Ct represents the cycle number at which the fluorescence intensity increased significantly above the background. The relative amount of PUUV RNA measured in the 3 virus stocks was standardized against the amount of HTNV RNA added to the samples. Recovery varied by about $30 \%$ according to the amount of quantified HTNV RNA which was spiked.

\section{Western Blot Analysis}

For Western blot analysis, proteins from $300 \mu \mathrm{l}$ of each virus were precipitated with trichloroacetic acid (final concentration $4 \%$ ) for $10 \mathrm{~min}$ at room temperature. Samples were centrifuged at $15,000 \mathrm{~g}$ for $5 \mathrm{~min}$ and proteins were resuspended in sample puffer (Tris-HCl 250 mM, SDS 70 mM, glycerol 10\%, mercaptoethanol $5 \%$, bromophenol blue $0.01 \%$ ). Ten percent of the samples were separated by $10 \%$ SDS-PAGE and blotted onto nitrocellulose membranes (Whatman $\mathrm{GmbH}$, Dassel, Germany). N protein was detected with sera from a rabbit immunized with recombinant $\mathrm{N}$ protein from PUUV and HTNV [9]. Signals were visualized by 
Table 1. Effect of the total amount of defective and infectious particles on FRNT results

\begin{tabular}{llll}
\hline & $\begin{array}{l}\text { PUUV- } \\
\text { low }\end{array}$ & $\begin{array}{l}\text { PUUV- } \\
\text { medium }\end{array}$ & $\begin{array}{l}\text { PUUV- } \\
\text { high }\end{array}$ \\
\hline $\mathrm{FFU/ml^{ \textrm {a } }}$ & $2.5 \times 10^{4}$ & $1 \times 10^{5}$ & $2.5 \times 10^{6}$ \\
Infectivity $_{\mathrm{N} \text { protein levels }}^{\mathrm{b}}$ & 1 & 4.0 & 100 \\
RNA levels & 1 & 2.8 & 19.6 \\
Infectivity/N protein & 1 & 1.9 & 10.5 \\
Infectivity/PUUV RNA & 1 & 1.4 & 5.1 \\
\hline
\end{tabular}

Reciprocal neutralizing anti-

body titer $^{c} \quad 160 / 160 \quad 160 / 320 \quad 640 / 640$

The calculated ratio between infectivity and virus particles is based on the determined amount of infectious virions, $\mathrm{N}$ protein and RNA in the PUUV stocks.

a The amount of infectious virus was titrated in Vero cells.

${ }^{\mathrm{b}}$ Relative amounts of $\mathrm{N}$ protein and RNA were determined by Western blot and quantitative RT-PCR, respectively.

${ }^{\mathrm{C}}$ The reciprocal neutralising antibody titer sufficient to reduce infectivity below $80 \%$ was determined by FRNT. Results of 2 independent experiments are shown.

The amounts of infectious particles (infectivity), $\mathrm{N}$ protein, PUUV RNA and neutralizing antibody titer in stock PUUV-low were arbitrarily defined as 1 . The ratio between infectivity and $\mathrm{N}$ protein and viral RNA, respectively, was used as a marker for the amount of defective particles in the stocks.

enhanced chemiluminescence (Pierce/Perbio Science, Bonn, Germany) and quantified densitometrically with TINA 2.0 software (Raytest, Straubenhardt, Germany).

\section{Focus Reduction Neutralization Test}

FRNT was performed as described previously [4]. Briefly, serial dilutions of a convalescent serum from a PUUV-infected patient were incubated for $1 \mathrm{~h}$ at $37^{\circ}$ with $50 \mathrm{FFU}$ PUUV. Thereafter, virus/serum mixtures were added to confluent Vero cells and incubated for $1 \mathrm{~h}$ at $37^{\circ}$. Cells were fed with medium containing $0.5 \%$ agarose and incubated for 10 days at $37^{\circ}$. Infectivity of the samples was quantified as described above. The titer of neutralizing antibodies reflects the reciprocal dilution of the serum sufficient to reduce infectivity by more than 5 -fold, compared to the virus control without serum.

\section{Results and Discussion}

Recently, we established a method for cloning noncytolytic viruses. This procedure was applied to a huge set of different hantaviruses and generally resulted in a pronounced increased yield of infectious virus [8]. This in-

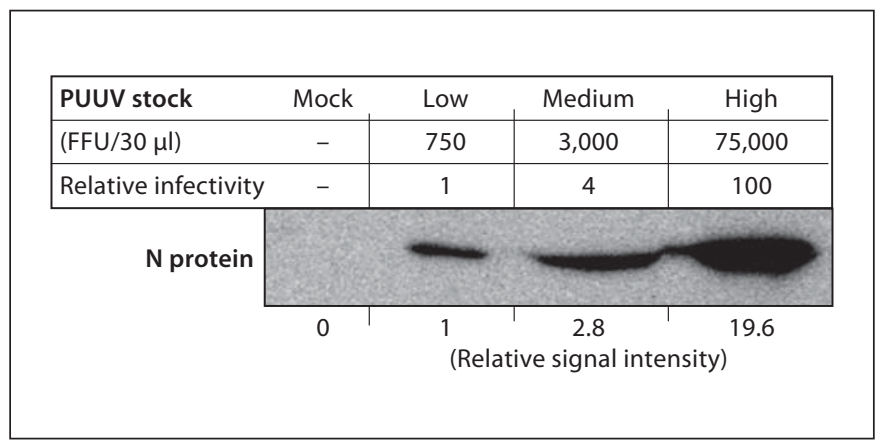

Fig. 1. The amount of $\mathrm{N}$ protein does not correlate with the amount of infectious particles in 3 different virus stocks. Aliquots (30 $\mu$ l each) from PUUV stocks with different virus titers (FFU) per milliliter were precipitated with trichloroacetic acid. The relative arbitrary amount of PUUV N protein in the 3 stocks was determined by Western blot analysis.

crease could be caused by the removal of DPs or defective interfering particles, which are produced at the expense of infectious particles. Thus, stocks with lower virus titers before focus purification should contain more DPs relative to the amount of infectious particles than stocks with higher titers derived from focus purified material.

To test the latter assumption, we compared $\mathrm{N}$ protein levels and infectivity in stocks produced before and after focus purification. The amount of the structural N protein should reflect the level of both defective and infectious virus particles and be consistent to the theory that the ratio between infectivity and $\mathrm{N}$ protein levels should increase with the virus titer. The stocks were designated PUUV-low, PUUV-medium and PUUV-high according to the titer with $2.5 \times 10^{4}, 1 \times 10^{5}$ and $2.5 \times 10^{6} \mathrm{FFU} /$ $\mathrm{ml}$, respectively. PUUV-medium and PUUV-high stocks were derived from virus material which had been focuspurified 1 and 3 times, respectively. PUUV-low was not subjected to this procedure. N protein levels of the stocks were determined by Western blot analysis after precipitation of proteins with trichloroacetic acid. Based on the input (30 $\mu$ l each), the equivalent of $750,3,000$, and 75,000 infectious virions of the stocks PUUV-low, PUUV-medium and PUUV-high, respectively, were loaded (fig. 1). The intensity of the N protein-specific signals detected did not correlate with the amount of infectious virions applied. As calculated and presented in table 1, PUUVmedium stock had 4-fold higher infectivity and about 2.8-fold higher $\mathrm{N}$ protein levels compared to PUUV-low stock. The PUUV-high stock, with 100-fold higher infec- 
tivity, had only about 20 -fold higher $\mathrm{N}$ protein levels than PUUV-low stock. These data show that the ratio between infectivity and $\mathrm{N}$ protein levels increased with the titer of the stocks. Based on these data, the stock with the highest virus titer contained about 5 -fold fewer DPs per infectious particle compared to the stock with the lowest titer.

There are indications that hantaviruses cause apoptosis in Vero cells $[2,10]$. Therefore, $\mathrm{N}$ protein not associated with particles might also be present in the culture medium released from dying cells. Assuming that such an apoptotic effect should be more pronounced in cells producing more viral products, the amount of $\mathrm{N}$ protein not associated with particles should correlate with the virus titers of the stock. If this potential scenario takes place, the enrichment of infectious versus defective particles by repeated focus purification should actually be even higher than the calculated factor.

Next, viral RNA was quantified, which can also be used to calculate the amount of both types of particles in the stocks. PUUV-low stock contained 4- and 100-fold fewer infectious particles compared to PUUV-medium and PUUV-high stocks, respectively. Compared to infectivity, the relative amount of PUUV RNA was only 2- and 10 -fold higher in the corresponding stocks. These data suggest that the stock with the highest virus titer contained about 10-fold fewer DPs per infectious particle compared to the stock with the lowest titer.

Tomato-spotted wilt virus (genus Tospovirus) and $\mathrm{Bu}-$ nyamwera virus (genus Orthobunyavirus), which belongs to the Bunyaviridae family, accumulate DPs with large internal genomic deletions $[11,12]$. In the presented study, the primers used for PCR were specific for the terminal region of the genomic L-segment close to the sites, which are thought to be essential for replication mediated by the viral RNA-dependent RNA polymerase [13]. Because accumulation of DPs requires replication of their defective genomes, at least part of the targeted regions should be present on both infectious and defective particles. Based on the data available, however, it cannot be excluded that the genomic defects of the RNA from DPs might, at least in part, impair PCR amplification with the selected primers. Therefore, the actual ratio between infectivity and RNA in stocks with high amount of DPs could be lower than calculated.

Taken together, our data demonstrate that the stock with the highest titer contained at least 5 -fold (based on the amount of $\mathrm{N}$ protein), but not more than 10 -fold (based on amount of PUUV RNA), fewer DPs per infectious particle compared to the stock with the lowest titer.
Irrespective of the presence or absence of DPs, it cannot be excluded that increased titers observed after focus purification might also be caused by direct adaptation of the selected virus clones to the cell culture system.

The 3 stocks containing high, medium and low relative amounts of DPs were used to quantify neutralizing antibodies by FRNT in a serum from a convalescent patient, who had been infected with PUUV. The reciprocal serum dilution sufficient to block $80 \%$ of the infectivity is shown as the neutralizing antibody titer in table 1 . Interestingly, the FRNT results varied depending on the virus stock used. The observed FRNT titer was 4 -fold higher when determined with the PUUV-high stock than with PUUV-low stock. The measured antibody titers inversely correlated with the amount of DPs and directly with the ratio between infectivity and $\mathrm{N}$ protein levels of the stocks. These data indicate that higher amounts of DPs in virus stocks yielded lower antibody titers by FRNT. The correlation supports our hypothesis that varying amounts of DPs can modulate results of virus neutralization tests.

Taken together, our data indicate for the first time that high levels of DPs in virus stocks used for neutralization assays lead to an underestimation of the amount of neutralizing antibodies. The study demonstrates that the amount of DPs in reference stocks should be considered when the amount of hantavirus-specific neutralizing antibodies is determined by FRNT. This observation might be important not only for diagnosis of hantavirus infections, but also for other assays in which reference virus stocks are used to quantify neutralizing antibodies in vitro.

\section{Acknowledgements}

We thank Renate Franke, Brigitte Pohl and Christina Priemer for their excellent technical assistance. We appreciate the helpful discussions with Paul-Ruben Marali and Martin Raftery. This work was supported by the Deutsche Forschungsgemeinschaft (RA-1057/2-1). 


\section{References}

1 Schmaljohn C, Hjelle B: Hantaviruses: a global disease problem. Emerg Infect Dis 1997;3:95-104.

-2 Schönrich G, Rang A, Lutteke N, Raftery MJ, Charbonnel N, Ulrich RG: Hantavirus-induced immunity in rodent reservoirs and humans. Immunol Rev 2008;225:163-189.

3 Bunyaviridae; in Fauquet CM, Mayo MA, Maniloff J, Desselberger U, Ball LA (eds): Virus Taxonomy: Classification and Nomenclature of Viruses. Eighth Report of the Committee on the Taxonomy of Viruses. Elsevier Academic Press, Amsterdam, 2005.

$\checkmark 4$ Heider H, Ziaja B, Priemer C, Lundkvist A, Neyts J, Krüger DH, Ulrich R: A chemiluminescence detection method of hantaviral antigens in neutralisation assays and inhibitor studies. J Virol Methods 2001;96:17-23.
5 Niklasson B, Jonsson M, Lundkvist A, Horling J, Tkachenko E: Comparison of European isolates of viruses causing hemorrhagic fever with renal syndrome by a neutralization test. Am J Trop Med Hyg 1991;45:660 665.

6 Bangham CR, Kirkwood TB: Defective interfering particles and virus evolution. Trends Microbiol 1993;1:260-264.

7 Kirkwood TB, Bangham CR: Cycles, chaos and evolution in virus cultures: a model of defective interfering particles. Proc Natl Acad Sci USA 1994;91:8685-8689.

-8 Rang A, Heider H, Ulrich R, Krüger DH: A novel method for cloning of non-cytolytic viruses. J Virol Methods 2006;135:26-31.

$\checkmark 9$ Razanskiene A, Schmidt J, Geldmacher A, Ritzi A, Niedrig M, Lundkvist A, Krüger DH, Meisel H, Sasnauskas K, Ulrich R: High yields of stable and highly pure nucleocapsid proteins of different hantaviruses can be generated in the yeast Saccharomyces cerevisiae. J Biotechnol 2004;111:319-333.
10 Hardestam J, Klingstrom J, Mattsson K, Lundkvist A: HFRS causing hantaviruses do not induce apoptosis in confluent Vero E6 and A-549 cells. J Med Virol 2005;76:234240 .

11 Patel AH, Elliott RM: Characterization of Bunyamwera virus defective interfering particles. J Gen Virol 1992;73:389-396.

$>12$ Resende Rde O, de Haan P, van de Vossen E, de Avila AC, Goldbach R, Peters D: Defective interfering L RNA segments of tomato spotted wilt virus retain both virus genome termini and have extensive internal deletions. J Gen Virol 1992;73:2509-2516.

13 Elliott RM, Schmaljohn CS, Collett MS: Bunyaviridae genome structure and gene expression. Curr Top Microbiol Immunol 1991; 169:91-141. 